\title{
Self-Organized Criticality Induced by Diversity
}

\author{
Álvaro Corral, Conrad J. Pérez, and Albert Díaz-Guilera \\ Departament de Física Fonamental, Facultat de Física, Universitat de Barcelona, Diagonal 647, E-08028 Barcelona, Spain
} (Received 14 August 1996)

\begin{abstract}
We have studied the collective behavior of a population of integrate-and-fire oscillators. We show that diversity, introduced in terms of a random distribution of natural periods, is the mechanism that permits one to observe self-organized criticality (SOC) in the long time regime. As diversity increases the system undergoes several transitions from a supercritical regime to a subcritical one, crossing the SOC region. Although there are resemblances with percolation, we give proofs that criticality takes place for a wide range of values of the control parameter instead of a single value. [S0031-9007(97)02469-1]
\end{abstract}

PACS numbers: 64.60.Lx, 87.10.+e, 64.60.Ak

In spite of the great interest received during the last decade by many systems exhibiting self-organized criticality (SOC) [1], it is still an open question to find necessary or sufficient conditions to observe this phenomenon, since there is no framework to predict, a priori, whether an arbitrary extended system will be, by its own dynamics and without any parameter tuning, critical in the long time regime.

Nevertheless, there is a set of common trends which characterize systems displaying SOC [2]. One of them concerns the dynamics that drives the elements of the system to a certain threshold. When some unit reaches the threshold, interaction between elements takes place, triggering a chain process or avalanche that ends when all the elements are below the threshold again. Then, a power-law distribution of avalanche sizes is the hallmark of SOC. A key point, crucial to observe SOC, is the separation between the slow time scale associated with the process that leads the units to the threshold (driving), and the fast time scale associated with the interaction (avalanches). Conservation was also believed essential to obtain SOC. Certainly, for the sandpile model [1] and other randomly driven models it is an indispensable requirement. A nonconservative dynamics introduces a characteristic length independent of system size [3]. However, several continuously driven models proposed later changed the widespread belief [4]. In these models SOC is not necessarily destroyed in a nonconservative regime and the distribution of avalanche sizes follows a power-law decay in a wide region of parameter space, with exponents depending on the level of dissipation.

Another point not studied so profoundly concerns the individual features of each element in the system. Up to now, it has been common to assume that all the units are identical. However, if SOC should have any relevance in physics or biology it should be robust in spite of the inherent differences between the members of a population. In other words, diversity should not destroy the critical properties of a given self-organized system. The object of this paper is to show that diversity not only does not break SOC but, as a matter of fact, it is the mechanism that enables one to observe it for certain continuously driven models which do not exhibit SOC under normal circumstances. The subject has a clear general interest. There are some collective phenomena such as the mutual synchronization of the members of a biological population [5] which traditionally have been tackled by assuming that all the units are identical. However, this assumption is not a necessary requirement. Several authors [6,7] have shown that after a suitable modeling, a group of nonidentical oscillators, each endowed with its own natural frequency, picked from a random distribution, may display a coherent temporal activity if the disorder level is below a certain critical value. A less intuitive opposite behavior has been also reported: disorder (diversity) can remove chaos and foster synchronization in a certain model of oscillators [8]. Uncorrelated differences between the members of the population trigger regular spatiotemporal patterns. In this paper we give evidence of another related phenomenon. A group of pulsecoupled oscillators evolve in a complex manner if they are identical, generating avalanches with many characteristic sizes (related with the linear dimension of the system). However, diversity will change the collective properties of the long time regime and will induce SOC.

Let us consider a population of integrate-and-fire oscillators. Each oscillator is defined in terms of a state variable $E$ which evolves in time as

$$
\frac{d E_{i}}{d t}=S-\gamma E_{i}
$$

and when $E_{i}$ reaches a threshold value $E_{\mathrm{th}}$, the $i$ th oscillator relaxes, and $E_{i}$ is redistributed instantaneously among its neighbors (labeled by $n$ ) according to

$$
\begin{gathered}
E_{i} \rightarrow 0 \\
E_{n} \rightarrow E_{n}+\varepsilon_{i n},
\end{gathered}
$$

and so on for every $E_{k} \geq E_{\mathrm{th}} \forall k$. This process, which continues until $E_{k}<E_{\mathrm{th}} \forall k$, constitutes an avalanche whose size $s$ is given by the number of relaxations (2). If 
$\sum_{n} \varepsilon_{i n} \leq E_{\mathrm{th}} \forall i$ except for at least one element for which the inequality is strict, it is guaranteed that avalanches of infinite size are impossible. Since the relaxing site is reset to zero and a fixed quantity $\varepsilon_{\text {in }}$ is transferred to the neighbors, the model is intrinsically nonconservative. It is assumed $\gamma E_{\mathrm{th}}<S$ and $\gamma$ is a non-negative constant whose physical meaning depends on the model one is dealing with. For instance, this model mimics a simplified version of the dynamics of spiking neurons, idealizing the cell membrane as an $R C$ circuit [9]. $E_{i}$ denotes the membrane potential of a given neuron, $\gamma^{-1}=R C$ the membrane time constant, $S$ (in appropriate units) a constant current that does act as a driving, and $\varepsilon_{\text {in }}$ the synaptic coupling strength between neurons $i$ and $n$. Equations (1) and (2) may also model the evolution of the cardiac pacemaker [10], swarms of flashing fireflies, and many other biological systems $[11,12]$.

In order to study local connectivity we have considered the case of a two-dimensional square lattice of linear size $L$ with nearest-neighbor uniform interactions, $\varepsilon_{\text {in }} \equiv$ $\varepsilon$. Recent studies on integrate-and-fire neurons [13] are more devoted to lattice models with periodic boundary conditions. However, the assumption of open boundary conditions breaks the homogeneous connectivity allowing the boundary units to be connected with less neighbors than the bulk units. This assumption will be present in the rest of the paper and makes the model also interesting in other fields. For $\gamma=0$ it reduces to a coupled map lattice proposed by Feder and Feder as a stick-slip model of earthquakes [14].

In addition, we have introduced diversity in terms of a random distribution of intrinsic periods. The period of each oscillator is given by

$$
T=\frac{1}{\gamma} \ln \left(\frac{S}{S-\gamma E_{\mathrm{th}}}\right) .
$$

There are different ways to introduce such types of quenched disorder in the model. One possibility is to assume a distribution of $R$ and $C$. Another option is to consider a distribution of input currents $S$. Both situations are plausible from a realistic point of view, but we have considered the latter. Let us mention that the most usual way to introduce diversity in this sort of model is by assuming a quenched random distribution of thresholds $[15,16]$. Although the distribution of thresholds also implies diversity in the intrinsic periods, it has influence in both the slow and the fast time scale, while our approach affects only the slow dynamics. This difference is crucial as we will see later.

When all the oscillators are identical the model described by (1) and (2) does not display SOC for any value of $\gamma \geq 0$ and $0<\varepsilon \leq 0.25$. Starting with uniformly distributed random initial conditions, $E_{i} \in\left[0, E_{\mathrm{th}} \equiv 1\right]$, for $\gamma=0$ and integer ratio $E_{\mathrm{th}} / \varepsilon$ only large avalanches take place because many units reach the threshold simultaneously $[17,18]$. If the ratio $E_{\mathrm{th}} / \varepsilon$ is not an integer, avalanches of all sizes are observed, but they are not power-law distributed [18]. For $\gamma>0$ (convex driving) the model exhibits a complex behavior which, depending on the particular values of $\gamma$ and $\varepsilon$ ranges from synchronization (in the sense that all the avalanches are exactly of size $L^{2}$ ) to events of all sizes distributed in a complicated way, as Fig. 1 illustrates. Here we observe that the probability density $P(s)$ for an avalanche of size $s$ presents a series of peaks at positions that are proportional to the linear size of the system $L$. This is a clear effect of the open boundaries. Moreover, the large peak of order $L^{2}$ confirms the tendency to synchronization for $\gamma>0$, which, however, in this case, the system is not able to sustain [19].

The situation changes completely for nonidentical oscillators. For simplicity we have considered a uniform distribution of periods. The width $\Delta$, expressed as the length of the symmetric interval $(T-\Delta / 2, T+\Delta / 2)$ centered without loss of generality around $T=1$, is a measure of disorder or diversity. In Fig. 2 we plot the distribution of avalanche sizes for different values of $\Delta$ for the same $\gamma$ and $\varepsilon$ as in Fig. 1. We observe several stages. First of all, the sequence of peaks displayed in Fig. 1 typical of identical oscillators continuously disappears when diversity increases. Then the distribution of avalanches becomes smoother, without intermediate peaks, but still maintaining the large one corresponding to avalanches of almost the size of the system $\left(L^{2}\right)$, as displayed for $\Delta=0.15$ in Fig. 2 where this trend towards synchronization can be seen clearly. The behavior is supercritical, because there are many events able to span the system. More interesting transitions take place as disorder increases. For a larger width, the system self-organizes in a critical state, without any spatial characteristic scale, as the power-law distribution of avalanche sizes in the curve with $\Delta=0.5$ of Fig. 2 indicates. The effect of the different periods is to reduce the probability of having large avalanches. Then, for very wide distributions of periods

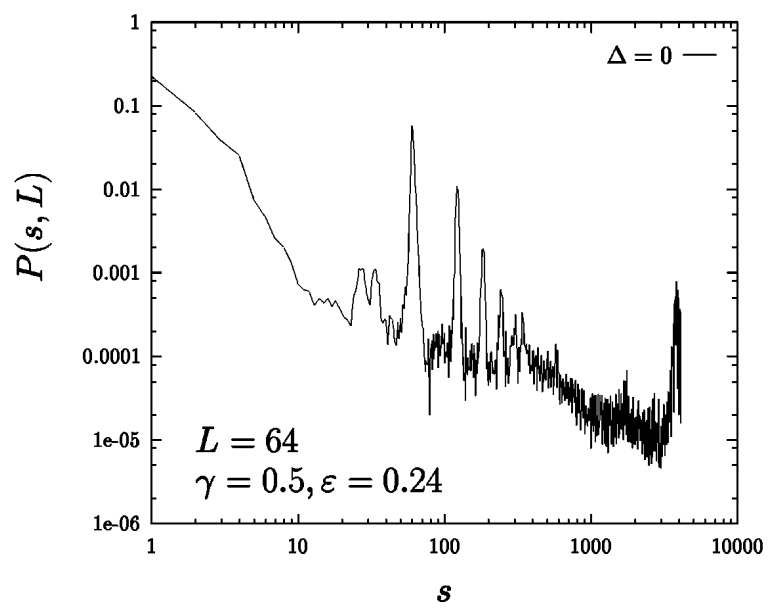

FIG. 1. Log-log plot of the stationary distribution of avalanche sizes $P(s)$ versus $s$ for the model without diversity. 


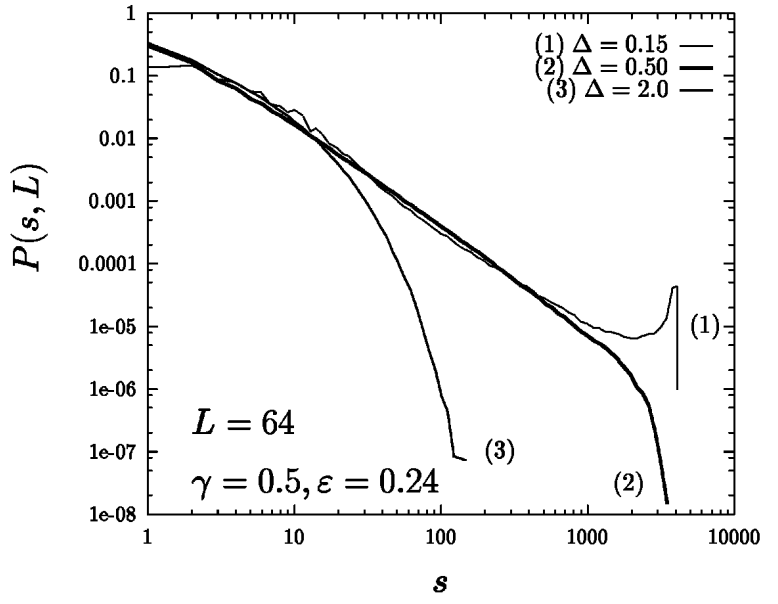

FIG. 2. Same as Fig. 1 for different degrees of diversity.

one could expect a strong decay of $P(s)$. In fact, this is what happens when $\Delta \geq 1.5$ for the case of Fig. 2, as exemplified by the curve with $\Delta=2$ : a characteristic scale independent of the system size appears and is responsible for the exponential decay. This means that for large diversity avalanches are localized and the system is subcritical. These transitions are not sharp, and the reported values of $\Delta$ can change with $\varepsilon$ and $\gamma$. In particular the loss of criticality and the appearance of a finite correlation length have been found difficult to characterize. Notice the resemblance between the three curves in Fig. 2 and those found in percolation, where the critical region is restricted to a single point of the control parameter [20]. However, our model shows a finite region of criticality instead of an infinitesimal one, as we are going to show below. This kind of behavior is, for the best of our knowledge, the first case where SOC is found between a supercritical region and a subcritical one in this class of models.

Let us pay some attention to the region where the power-law decay of $P(s)$ is reported. We have performed a finite-size scaling analysis for different widths $\Delta$. The results are shown in Fig. 3. A data collapse for different system sizes $L$ is obtained when plotting $L^{\beta} P(s, L)$ against the rescaled variable $s / L^{\nu}$. The increment of system size does not show any deviation from the scaling for separate enough values of the control parameter $\Delta$, supporting our statement of a critical region instead of a critical point. As a complement we plot in Fig. 4 the mean size of the avalanches as a function of system size, for different values of diversity. The behavior $\langle s\rangle \sim$ $L^{2 \nu-\beta}$ (consequence of the scaling ansatz) even for large $L$ confirms the scaling in the critical region. In addition, we have released the restriction of identical coupling strengths, introducing randomness in space $\left(\varepsilon_{i j}\right.$ quenched random variable) or in space and time $\left[\varepsilon_{i j}(t)\right.$ annealed], by means of a uniform random distribution around the mean value $\varepsilon$. We have verified that SOC is robust under

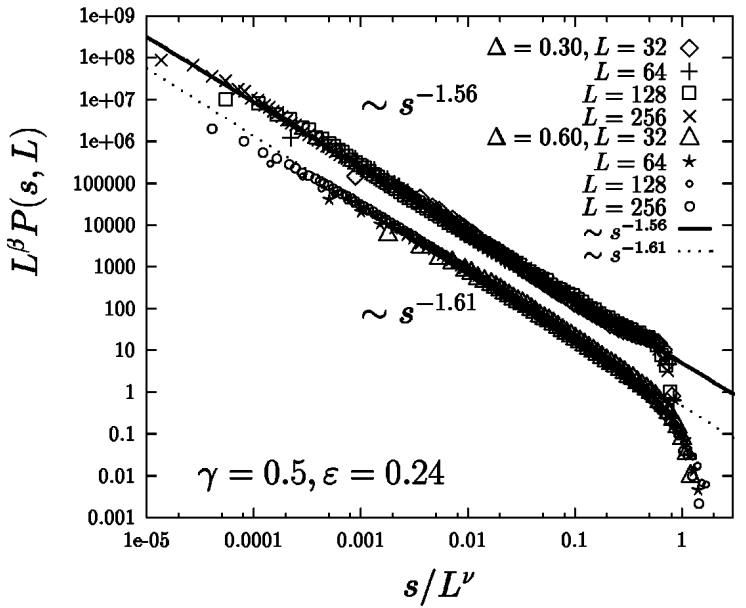

FIG. 3. Finite-size scaling analysis of the distribution of avalanche sizes for the critical region. For $\Delta=0.3$ we obtain $\nu=2.0$ and $\beta=3.15$ (this curve has been shifted one decade upwards for clarity sake), whereas for $\Delta=0.6, \nu=1.8$ and $\beta=2.85$ are used.

this perturbation and hence identical couplings are not a necessary condition to obtain criticality. This feature could be relevant in realistic models of spiking neurons.

The results shown so far are not characteristic of a particular value of the parameters which describe the system. In fact there is a region in the $(\gamma, \varepsilon)$ space where diversity induces SOC and it corresponds to large values of $\varepsilon$ and small $\gamma$. It would be very interesting to have knowledge of the complete phase diagram of the model.

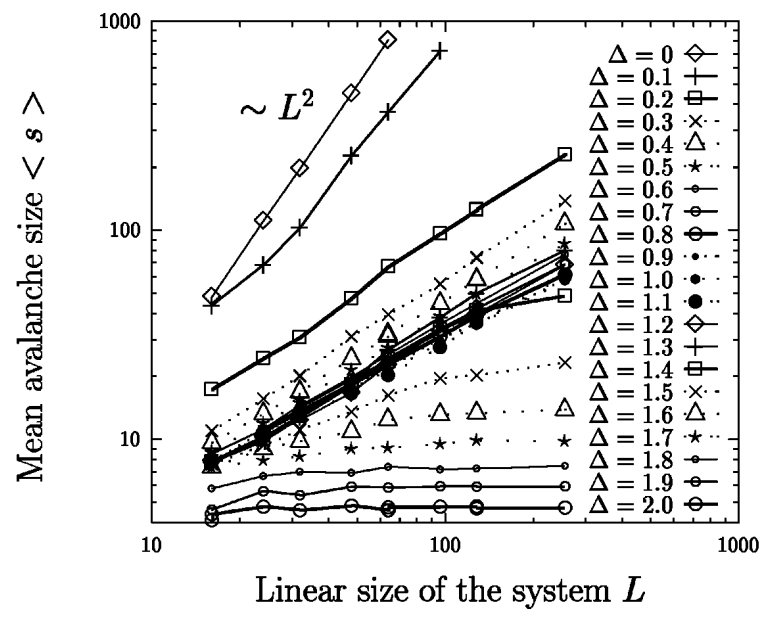

FIG. 4. Mean size of the avalanches as a function of the system size for different values of diversity using $\gamma=$ $0.5, \varepsilon=0.24$. For identical units $\langle s\rangle$ scales as $L^{2}$. This exponent decreases continuously with increasing diversity in the supercritical region. Notice the accumulation of data points for a wide range of $\Delta$ values (starting for $\Delta \geq 0.3$ ) in a narrow interval of mean avalanche sizes, corresponding to the critical region. Here the data fit the scaling $L^{2 \nu-\beta}$. It is difficult to predict up to which values the scaling holds, but when $\Delta \geq 1.5$ the growth of $\langle s\rangle$ with $L$ is clearly logarithmic, in agreement with a subcritical region. 
However, taking into account that three parameters are involved, $\gamma, \varepsilon$, and the width $\Delta$, and different system sizes are needed, a complete sweep of the phase space would require an enormous effort, which is beyond our possibilities. Nevertheless, let us mention that for large $\gamma$ there exists a range of $\varepsilon$ values which give complete synchronization, in the sense previously explained, no matter the width of the distribution of periods.

Our results also have sense in the context of earthquakes if we imagine the Feder and Feder model as a rough version of the Burridge-Knopoff spring-block model [21]. The different intrinsic periods of each unit will be caused by different elastic constants in the springs connecting the blocks with the driving plate. When $\gamma>0$ a nonlinearity in the elastic response of these springs is introduced. With the same goal in mind we have examined our disordered model replacing (2) by the Olami et al. (OFC) rules [4,19], and we have found that the SOC region is robust in spite of a very large disorder, although eventually it can give rise to localized avalanches. These results contrast with the studies performed in Refs. $[15,16]$ where a distribution of thresholds was considered. It was found that while disorder is irrelevant in the conservative regime, it destroys criticality for the dissipative case, leading to an exponential distribution of avalanche sizes [15]. A similar change in the collective properties of the disordered system has been used to claim the lack of robustness of OFC as a model of earthquakes [16]. Other authors [22] have considered the influence of defects in the model. The main result was to observe that SOC is robust even for a large number of defects. Notice that for the same model randomness included in different parameters leads to different behaviors.

Finally, let us remark on the effect that different types of noise may have on the collective features of the model. The original properties of the Feder and Feder model (with $\gamma=0$ ) are not robust to noise, e.g., altering the relaxation rule (2) by adding a small random number to any reset unit changes the cooperative behavior of the system $[17,18]$. It does not tend to form a few groups of elements with the same phase, but it goes towards a SOC state. Note that this type of noise has a completely different nature than the quenched source of diversity considered in this paper. While the first can be triggered by internal fluctuations, the second is an inherent feature of each member of the population. Furthermore, while the dynamic noise only induces SOC in the linear regime $(\gamma=0)$, and for very small noise intensities, diversity induces SOC in a wide region of the parameter space.

In summary, we give an example showing that diversity is a new mechanism for the emergence of SOC and that criticality in nonequilibrium systems is not just a singularity in parameter space, as it happens in equilibrium. Our results have interest for models of integrate-and-fire neurons as well as for earthquakes.
We are indebted to A. Arenas, P. Bak, K. Christensen, and B. Tadić for many suggestions and discussions. A.C. acknowledges the warm hospitality of Brookhaven National Laboratory as well as a scholarship of the Spanish MEC. This work has been supported by CICyT under Grant No. PB94-0891.

Note added.- Just after submitting this paper we became aware of Ref. [23] where disorder is introduced in the couplings for the OFC model (with $\gamma=0$ ), attaining a collective behavior in agreement with our results.

[1] P. Bak, C. Tang, and K. Wiesenfeld, Phys. Rev. Lett. 59, 381 (1987); Phys. Rev. A 38, 364 (1988).

[2] We are not considering extremal models; for a review, see M. Paczuski, S. Maslov, and P. Bak, Phys. Rev. E 53, 414 (1996).

[3] S. S. Manna, L. B. Kiss, and J. Kertész, J. Stat. Phys. 61, 923 (1990).

[4] Z. Olami, H. J. S. Feder, and K. Christensen, Phys. Rev. Lett. 68, 1244 (1992); K. Christensen and Z. Olami, Phys. Rev. A 46, 1829 (1992).

[5] J. Buck and E. Buck, Sci. Am. 234, 74 (1976).

[6] A.T. Winfree, The Geometry of Biological Time (Springer, New York, 1980).

[7] Y. Kuramoto, Chemical Oscillations, Waves and Turbulence (Springer-Verlag, Berlin, 1984).

[8] Y. Braiman, J. F. Lindner, and W. L. Ditto, Nature (London) 378, 465 (1995); S. H. Strogatz, Nature (London) 378, 444 (1995).

[9] H. C. Tuckwell, Introduction to Theoretical Neurobiology (Cambridge University Press, Cambridge, 1988).

[10] C.S. Peskin, Mathematical Aspects of Heart Physiology (Courant Institute of Mathematical Sciences, New York University, New York, 1975).

[11] R. E. Mirollo and S. H. Strogatz, SIAM J. Appl. Math. 50, 1645 (1990).

[12] A. Corral, C. J. Pérez, A. Díaz-Guilera, and A. Arenas, Phys. Rev. Lett. 75, 3697 (1995).

[13] J. J. Hopfield and A. V. M. Herz, Proc. Natl. Am. Soc. USA 92, 6655 (1995).

[14] H. J.S. Feder and J. Feder, Phys. Rev. Lett. 66, 2669 (1991).

[15] I. M. Janosi and J. Kertesz, Physica (Amsterdam) 200A, 179 (1993).

[16] F. Torvund and J. Froyland, Phys. Scr. 56, 642 (1995).

[17] K. Christensen, Ph.D. thesis, University of Aarhus, Denmark, 1992.

[18] C. J. Pérez, A. Corral, A. Díaz-Guilera, K. Christensen, and A. Arenas, Int. J. Mod. Phys. B 10, 1111 (1996).

[19] A. Corral, C. J. Pérez, A. Díaz-Guilera, and A. Arenas, Phys. Rev. Lett. 74, 118 (1995).

[20] D. Stauffer and A. Aharony, Introduction to Percolation Theory (Taylor and Francis, London, 1994).

[21] R. Burridge and L. Knopoff, Bull. Seismol. Soc. Am. 57, 341 (1967).

[22] H. Ceva, Phys. Rev. E 52, 154 (1995).

[23] N. Mousseau, Phys. Rev. Lett. 77, 968 (1996). 